\title{
DIVERSITY AND STATUS OF BUTTERFLIES IN LOWLAND DISTRICTS OF WEST NEPAL
}

\author{
B. Khanal
}

\begin{abstract}
This paper is an outcome of the studies made in four districts (Dangdeukhuri, Banke, Bardia and Surkhet) of western Terai in different months of various years. Altogether 85 species categorized into 64 genera and 10 families have been reported with their status categories based on national status list. The process of urbanization has altered valuable natural habitats of many of the rare species especially in Banke and Dangdeukhuri districts. Monitoring process under the established regulation and thorough investigations in many unexplored areas of this region have been felt essential so to design suitable conservation measures to butterflies of this region.
\end{abstract}

Key words: Biodiversity, western terai, butterflies, unexplored, habitats.

\section{Introduction}

Among the covered districts, Dang valley in Dangdeukhuri district is the inner Terai bounded around by Mahabharat and Siwalik mountains to the north and south respectively. The remaining districts of the lower Terai like Surkhet, Banke and Bardia are under complete influence of the subtropical to tropical climatic types. These districts once were very popular for displaying remarkable luxuriant forests are now heavily pressurized under human activities mainly for settlements, agricultural practices, and urbanization. These districts are characterized with their own river systems which include big rivers like Rapti in Dangdeukhuri and Banke, Bheri in Surkhet and Karnali in Bardia.

Forests in west lowlands are mainly deciduous or mixed deciduous types. The mesic riverine forest shelters rich and diverse butterflies compared to the dry areas. The popular vegetation components seen in these parts are Shorea robusta, Michelia spp., Ficus spp., Bamboo, Dalbergia sisoo etc. The altitudinal variation in these districts ranges from $129 \mathrm{~m}$ to $2060 \mathrm{~m}$ (Anonymous, 1971).

Comparatively, Bardia still has retained good forested zones including the well known Bardia National Park which is worth to safeguarding many of the rare flora and fauna occurring here. Rare fauna in this park include Bengal Tiger (Panthera tigris), One Horned Rhinoceros (Rhinos unicornis), Asiatic Elephant (Elephas maximus) and Gharial (Gavialis gangeticus). The Siwalik areas of Dang and Deukhuri exhibit many valuable fossil elements like the skull of Archidiskodon planifrons, an extinct elephant fossil, and many petrified bone remnants of crocodiles and other animals.

A change in butterfly diversity is an interesting phenomenon noticed in this region owing to its land structure and changing vegetation pattern within a short stretch of Dang to Surkhet Valleys. The butterfly diversity observed here is more diversified around the riverine forests. 
The National Park in Bardia shelters rich diversity while deforested zones of Banke and Surkhet are mostly furnished with common and robust species. Many parts of Bardia still retain potential habitats to many of the rare species.

The influence of warm climate and occurrence of diverse floral components have widened butterfly season in these areas. The said suitable season generally starts here from March to mid-November. Many interesting and rare species emerge out in May till August. Some robust species which have ability to tolerate cold climatic condition still can be seen till last of December. Species like Cabbage White, Peacock Pansy, Tortoise Shell, Grass Yellows etc. occur here year round.

\section{Materials and Methods}

Studies conducted in different periods from 1988 to 1993 and 1999 to 2003 have been incorporated in this paper. Only those species of butterflies not promptly identifiable in the field were collected by butterfly net. Notes on complete field data of each observed and collected species included location altitude, collection date, collection locality and assessed species status based on national status scale. Collected specimens were identified at the Natural History Museum which comprised the process of tallying museum specimens and consultations of characteristic keys developed by Talbot (1975). All the collected specimens are deposited at the Natural History Museum in Kathmandu.

\section{Result}

All the reported butterflies with their scientific names, altitudinal range and current status are provided below in tabulated form. The status of every observed species was determined by their frequencies in the field, consulting Smith (1989) and checking Nepal Red Data Book (NRDB) Status Data (1995). Many species in this study were common excluding few like Euploeopsis clytia f. dissimilis , Catopsilia pomona f. catilla Fabricius, Chilades pandava, Tarucus callinara, Curetis dentata, Rapala manea schistacea, Spindasis elima uniformis, Horaga onyx, Rapala nissa, Remelana jangala and Thoressa aina (Table 1) which were specific to their distributional range and occurred in certain localities only. Few species like Papilio demoleus, Menelaides polytes, Eurema hecabe, Pieris brassicae nepalensis, Pieris canidia indica, Catopsilia pyranthe pyranthe, Heliophorous epicles, Zizeeria maha maha, Freyeria putli, Pantoporia hordonia, Precis orithya ocyale, Precis hierta, Precis almana almanac, Precis iphita, Vanessa indica indica, Precis lemonias persicaria, , Neptis hylas, Melanitis leda leda, Mycalesis persius blassius, Orsotrioena medus, Tirmala limniace leopardus,, Danaus chryssipus chryssipus, Danaus genutia and Euploea core core were quite prevalent and shared their habitats in all the four districts of west Terai (Table 1). Majority of the species reported here were fond of open field, riverine forests, cultivated lands and forested areas. 
Table 1. Summary results of the status of butterfly fauna in districts of Dangdeukhuri, Banke, Bardia and Surkhet is provided below in tabulated form. This list is based upon the study made in various periods during 1988 to 2003.

\begin{tabular}{|c|c|c|c|c|c|c|}
\hline \multirow[t]{2}{*}{ Genus and species } & \multicolumn{4}{|c|}{ District } & \multirow{2}{*}{$\begin{array}{l}\text { Altitude } \\
(\mathrm{m})\end{array}$} & \multirow[t]{2}{*}{ Status } \\
\hline & Dangdeukhuri & Banke & Bardia & Surkhet & & \\
\hline $\begin{array}{l}\text { PAPILIONIDAE } \\
\text { Menelaides nephelus chaon } \\
\text { Westwood }\end{array}$ & + & - & - & - & 160 & Uncommon \\
\hline Menelaides polytes Linnaeus & + & + & + & + & Every-where & Common \\
\hline Iliades memnon Linnaeus & + & - & - & - & 1100 & Uncommon \\
\hline $\begin{array}{l}\text { Euploeopsis clytia f. dissimilis } \\
\text { Linnaeus }\end{array}$ & - & - & - & + & 400 & Rare \\
\hline Papilio demoleus Linnaeus & + & + & + & + & $200-400$ & Common \\
\hline Pachliopta aristolochiae Fabricius & + & - & - & - & 450 & Common \\
\hline Deoris nomius Esper & + & + & - & - & $300-450$ & Uncommon \\
\hline $\begin{array}{c}\text { PIERIDAE } \\
\text { Metaporia agathon Gray }\end{array}$ & + & - & - & - & 2820 & Common \\
\hline Eurema hecabe Linnaeus & + & + & + & + & $\begin{array}{l}\text { Various } \\
\text { altitudes }\end{array}$ & Common \\
\hline Eurema laeta Boisduval & + & - & - & + & 200-910 & Uncommon \\
\hline $\begin{array}{l}\text { Pieris brassicae nepalensis } \\
\text { Doubleday }\end{array}$ & + & + & + & + & $\begin{array}{l}\text { Various } \\
\text { altitudes }\end{array}$ & Common \\
\hline Pieris canidia indica Evans & + & + & + & + & $\begin{array}{l}\text { Various } \\
\text { altitudes }\end{array}$ & Common \\
\hline $\begin{array}{l}\text { Catopsilia pyranthe pyranthe } \\
\text { Linnaeus }\end{array}$ & + & + & + & + & $200-800$ & Common \\
\hline Catopsilia pomona pomona Fabricius & - & + & - & + & $200-660$ & Uncommon \\
\hline Catopsilia pomona f. catilla Fabricius & + & - & - & - & & Rare \\
\hline Ixias pyrene familiaris Butler & + & - & - & + & $350-910$ & Common \\
\hline Belenois aurota aurota Fabricius & - & - & + & - & $200-500$ & Common \\
\hline Cepora nerissa phryne Fabricius & + & - & + & + & $250-900$ & Uncommon \\
\hline $\begin{array}{l}\text { LYCAENIDAE } \\
\text { Heliophorus sena Kollar }\end{array}$ & - & - & - & + & 785 & Uncommon \\
\hline Heliophorous epicles Godart & + & + & + & + & $200-900$ & Common \\
\hline Castalius rosimon Fabricius & + & + & + & - & $200-915$ & Common \\
\hline Chliaria othona Hewitson & + & - & - & - & 900 & Uncommon \\
\hline Anthene emolus emolus Godart & + & - & + & - & $500-848$ & Common \\
\hline Zizeeria maha maha Kollar & + & + & + & + & $200-900$ & Common \\
\hline Freyeria putli Kollar & + & + & + & + & $200-900$ & Common \\
\hline Zizeena otis otis Fabricius & + & - & + & - & $200-930$ & Uncommon \\
\hline Lampides boeticus Linnaeus & + & - & + & - & 300 above & Common \\
\hline Prosotas nora ardates Moore & + & - & + & + & $350-900$ & Common \\
\hline $\begin{array}{l}\text { Arhophala pseudocentaurus } \\
\text { Doubleday }\end{array}$ & - & + & + & - & $200-500$ & Common \\
\hline Euchrysops cnejus Fabricius & + & - & + & - & $200-500$ & Uncommon \\
\hline Chilades pandava Horsfield & - & - & - & + & 780 & Rare \\
\hline Freyeria trochilus orientalis Forster & - & - & - & + & 730 & Common \\
\hline Tarucus callinara Butler & - & - & - & + & 700 & Rare \\
\hline Curetis dentata Moore & - & - & + & + & $700-900$ & Rare \\
\hline Curetis bulis Westwood & + & - & + & + & $300-900$ & Uncommon \\
\hline Rapala manea schistacea Moore & - & - & + & + & $300-700$ & Rare \\
\hline Catochrysops strabo Fabricius & - & - & - & + & 700 & Uncommon \\
\hline Spindasis elima uniformis Moore & + & - & - & - & 850 & Rare \\
\hline Horaga onyx Moore & - & - & + & - & 340 & Rare \\
\hline
\end{tabular}


Khanal : Diversity \& status of..

\begin{tabular}{|c|c|c|c|c|c|c|}
\hline Rapala nissa Kollar & - & - & + & - & 320 & Rare \\
\hline Remelana jangala Horsfield & - & - & + & - & 165 & Rare \\
\hline $\begin{array}{l}\text { NYMPHALIDAE } \\
\text { Ariadne merione Cramer }\end{array}$ & + & - & + & - & $327-1090$ & Common \\
\hline Zemeros flegyas Cramer & + & - & + & + & $165-850$ & Common \\
\hline Cupha erymanthis lotis Sulzer & + & - & + & + & $160-930$ & Common \\
\hline Cyrestis thyodamus Boisduval & - & - & + & + & $202-700$ & Uncommon \\
\hline Hypolimnas bolina Linnaeus & + & - & + & + & $165-800$ & Common \\
\hline Symbrentia lilaea Hewitson & - & - & + & - & 350 & Common \\
\hline Pantoporia hordonia Stoll & + & + & + & + & $148-750$ & Common \\
\hline Precis orithya ocyale Hubner & + & + & + & + & $165-800$ & Common \\
\hline Precis hierta Fabricius & + & + & + & + & $170-859$ & Commom \\
\hline Precis almana almana Linnaeus & + & + & + & + & $200-800$ & Common \\
\hline Precis atlites Linnaeus & + & - & + & + & “ & " \\
\hline Precis iphita Cramer & + & + & + & + & $160-900$ & Common \\
\hline Phalanta phalantha Drury & + & - & - & + & $700-900$ & Common \\
\hline Vanessa indica indica Herbst & + & + & + & + & $200-900$ & Common \\
\hline Vagrans egista Cramer & + & - & + & + & $200-900$ & Common \\
\hline $\begin{array}{l}\text { Precis lemonias persicaria } \\
\text { Fruhstorfer }\end{array}$ & + & + & + & + & $200-900$ & Common \\
\hline Neptis hylas Linnaeus & + & + & + & + & $165-900$ & Common \\
\hline Athyma perius Linnaeus & + & - & + & + & 424- 850 & Common \\
\hline Kallima inachus Boisduval & + & - & + & + & $450-900$ & Uncommon \\
\hline $\begin{array}{c}\text { SATYRIDAE } \\
\text { Ypthima baldus baldus Fabricius }\end{array}$ & + & + & - & + & $350-900$ & Uncommon \\
\hline Ypthima singala Felder & + & + & + & - & 450 & Uncommon \\
\hline Ypthima huebneri Kirby & - & - & + & - & $166 \& 320$ & Uncommon \\
\hline Ypthima newara Moore & - & - & + & - & 410 & $\begin{array}{l}\text { Common } \\
\text { (ZSL 2003) }\end{array}$ \\
\hline Lethe confusa Aurivillius & - & - & - & + & 350 & Common \\
\hline Melanitis leda leda Linnaeus & + & + & + & + & $165-800$ & Common \\
\hline Mycalesis mineus Linnaeus & - & - & + & - & 202 & Common \\
\hline Mycalesis persius blassius Fabricius & + & + & + & + & $200-800$ & Common \\
\hline Orsotrioena medus Fabricius & + & + & + & + & $165-900$ & Common \\
\hline Elymnias hypermnestra Linnaeus & + & - & - & - & 900 & Uncommon \\
\hline $\begin{array}{c}\text { ACREIDAE } \\
\text { Acraea issoria Hubner }\end{array}$ & + & - & - & + & 300- 950 & Common \\
\hline $\begin{array}{l}\text { LIBYTHEIDAE } \\
\text { Libythea myrrha Godart }\end{array}$ & - & - & + & + & $220 \& 640$ & Common \\
\hline $\begin{array}{l}\text { NEMEOBIIDAE } \\
\text { Abisara bifasciata Moore }\end{array}$ & - & - & + & - & 448 & Common \\
\hline $\begin{array}{c}\text { DANAIDAE } \\
\text { Tirmala limniace leopardus Butler }\end{array}$ & + & + & + & + & $200-800$ & Common \\
\hline $\begin{array}{l}\text { Danaus chryssipus chryssipus } \\
\text { Linnaeus }\end{array}$ & + & + & + & + & $165-800$ & Common \\
\hline Danaus genutia Cramer & + & + & + & + & $200-900$ & Common \\
\hline Parantica aglea melanoides Moore & + & + & + & - & $180-900$ & Common \\
\hline Euploea core core Cramer & + & + & + & + & $165-900$ & Common \\
\hline Euploea mulciber mulciber Cramer & + & - & + & - & $200-900$ & Common \\
\hline Tirmala septentrionis Butler & - & - & + & - & 424 & uncommon \\
\hline $\begin{array}{r}\text { HESPERIIDAE } \\
\text { Thoressa aina DeNiceville }\end{array}$ & + & - & - & - & 600 & Rare \\
\hline Udaspes folus Cramer & + & + & - & - & $160 \& 730$ & Common \\
\hline Tagiades litigiosa Moschler & - & - & + & - & 450 & Common \\
\hline Badamia exclamationis Fabricius & - & + & - & - & 160 & Uncommon \\
\hline Pseudocoladenia dan Fabricius & + & - & + & + & $200-800$ & Common \\
\hline
\end{tabular}




\section{Discussion}

A diversity account of butterfly within the length of Dang to Surkhet valleys revealed out 85 species under 64 genera and 10 families, about 13\% of the total record (650 species) of the country hitherto. Majority of the species resulted in this study have affiliation to oriental elements characteristics of the tropical to subtropical climatic types. Popular species like Pieris brassicae nepalensis, Pieris indica, Aglais kashmirensis, Lampides boeticus etc. also share their habitats in the cold alpine zone besides the warmer habitats of lowland Terai. The basic approach to data updating is missing since 2003 mainly due to the lack of monitoring under the established guidelines. This study, though conducted for a short period, revealed an appreciable result especially at the valley floor and riverine ecosystem of Dangdeukhuri where 65 species were investigated in a short tenure of time. On district basis, Surkhet though exhibited diversified species but are mostly common and widely spread across nation. The localities especially the inner area of the Bardia National Park is remarkably interesting where multitude species of various status categories are under the safe line of protection. A brief survey over the Chepang Ghat of Bardia brought out a list of 78 species (ZSL, 2003) with none matching the protected list of the country (BPP, 1995). Some Nymphalids and Satyrids have remarkable display of seasonal variations. Most of the species observed in post autumn period display remarkable features of Dry Season forms (Khanal, 1999). Many of the significant and pristine habitats in this part are under demolishing state due to increasing pressure of humans, which definitely is changing the status of many species into the vulnerable state.

Among 11 families of butterflies occurring in Nepal, 10 families have been reported in this study except Amathusiidae which has just three species countrywide so far.

\section{Acknowledgement}

The Zoological Society of London and Natural History Museum in Kathmandu are specially acknowledged for providing me an opportunity to study butterflies of Bardia, west Nepal. I am thankful to the Nepal Academy of Science and Technology (NAST) for sponsoring me a small grant to study natural environment of Dang Valley in 1988. All colleagues at Natural History Museum who cooperated me in this work are specially acknowledged.

\section{References}

Anonymous. (1971). Paschimanchal. Mechi dekhi Mahakali, Part 3, Department of Information, Nepal Government, Kathmandu, Nepal.

BPP. (1995). Red Data Book of the Fauna of Nepal. Biodiversity Profile Project Technical Publication, No.4. Department of National Parks and Wildlife Conservation, Ministry of Forests and Soil Conservation, Nepal Government, Kathmandu:37-42.

Khanal, B. (1999). Checklist of butterflies from Kanchanpur and Kailali districts, far-west Nepal, Journal of Natural History Museum, Nepal, 18:61-69.

Smith, C. (1989). Butterflies of Nepal. Tecpress Service L.P., Bangkok, Thailand:32-267.

Talbot, G. (1975). Butterflies. Fauna of British India, Taylor and Francis, London:55-563. 
ZSL. (2003). Babai River Valley Fish and Biodiversity Survey. Royal Bardia National Park, Nepal (March 2003). The Zoological Society of London, v +119 pp. Oliver, S. (Ed.),

\section{Author's Address}

Bhaiya Khanal

Natural History Museum, Swayambhu

Kathmandu, Nepal.

Email: baya2000@hotmail.com 